\title{
Synthesis and Antimicrobial Evaluation of a New Series of $N$-1,3-Benzothiazol-2-ylbenzamides
}

\author{
Domenico Armenise, Alessia Carocci, Alessia Catalano, Marilena Muraglia, Ivana Defrenza, \\ Nicolino De Laurentis, Antonio Rosato, Filomena Corbo, and Carlo Franchini
}

Dipartimento di Farmacia-Scienze del Farmaco, Università degli Studi di Bari “Aldo Moro," Via E. Orabona 4, 70125 Bari, Italy

Correspondence should be addressed to Alessia Carocci; alessia.carocci@uniba.it and Alessia Catalano; alessia.catalano@uniba.it

Received 30 May 2013; Accepted 2 September 2013

Academic Editor: Gabriel Navarrete-Vazquez

Copyright (c) 2013 Domenico Armenise et al. This is an open access article distributed under the Creative Commons Attribution License, which permits unrestricted use, distribution, and reproduction in any medium, provided the original work is properly cited.

\begin{abstract}
Enterococcus faecalis is a Gram-positive commensal inhabitant of the intestinal tract of humans, animals, and insects. However, it is also an opportunistic pathogen and has emerged as a leading cause of hospital-acquired extraintestinal infections. Fluoroquinolones have been frequently used to treat $E$. faecalis infections, and the emergence of fluoroquinolone-resistant $E$. faecalis strains has recently been reported in several countries. Thus, the identifications of new antibiotics specifically directed to E. faecalis may be envisaged. In this paper, a new series of $N$-1,3-benzothiazol-2-ylbenzamides have been designed, synthesized, and evaluated for their in vitro antimicrobial activities. Among the tested compounds, $3 \mathbf{i}$ was active against $E$. faecalis.
\end{abstract}

\section{Introduction}

Drug resistance to therapeutic antibiotics poses a challenge to the identification of novel targets and drugs for the treatment of infectious diseases. Infections caused by Enterococcus faecalis are a major health problem. Thus, studies for the identification of novel targets and drugs for the treatment of infectious diseases are at the forefront. E. faecalis is a Grampositive opportunistic pathogen which has emerged as a leading cause of hospital-acquired extraintestinal infections, including urinary tract infections (UTIs), bacteremia, wound infections, and endocarditis [1-3]. Normally, a resident of the gastrointestinal tract, extensive use of antibiotics has resulted in the rise of E. faecalis strains that are resistant to multiple antibiotics. We recently determined the X-ray crystallographic structure of E. faecalis thymidylate synthase, which should be a potential target for antibacterial therapy [4]. Fluoroquinolones have been frequently used to treat $E$. faecalis UTIs, and the emergence of fluoroquinolone-resistant $E$. faecalis (QREF) strains has recently been reported in several countries [5]. Thus, the development of new and different antimicrobial drugs, in particular acting against $E$. faecalis, is a very important goal, and most of the research program efforts in this field are directed towards the design of new agents. During the past decade, combinatorial chemistry has provided access to chemical libraries based on privileged structures [6], with heterocyclic structures receiving special attention as they belong to a class of compounds with proven utility in medicinal chemistry [7-10]. Many heterocyclic nuclei, such as 1,3,4-thiadiazole [11], benzimidazole [12], 1,3,5-triazine [13], and benzothiazole [14], have been recently reviewed as antimicrobial agents. Our attention was focused on the benzothiazole nucleus. Benzothiazole derivatives possess a wide spectrum of biological applications such as antitumor [15-18], antimicrobial [19-21], schistosomicidal [22], antiinflammatory [23, 24], anticonvulsants [25-27], antidiabetic $[28,29]$, antipsychotic $[30,31]$, neuroprotective [32], and diuretic [33] activities. In the past, we were interested in a series of 2-mercapto-1,3-benzothiazole derivatives showing antimicrobial activity [19]. It is disclosed that the SH moiety at the $\mathrm{C} 2$ position of the heterocyclic nucleus led to a remarkable antibacterial activity against Gram positive and negative. Actually, in order to improve SAR studies on 1,3benzothiazoles, we decided to investigate the isosteric relationship between 2-mercapto and 2-amino substitutions. Moreover, in this study we also aimed at widening SAR 
<smiles>O=C(Nc1nc2cc(F)ccc2s1)c1ccc(F)cc1</smiles>

1<smiles>O=C(Nc1nc2cc(F)ccc2s1)c1ccc(F)cc1</smiles>

3

FIGURE 1<smiles>Nc1nc2cc(F)ccc2s1</smiles>

2<smiles>Nc1nc2cc(F)ccc2s1</smiles>

5
TABLE 1: Structures of compounds $\mathbf{3 a}-\mathbf{l}$ and $\mathbf{5 a} \mathbf{a} \mathbf{b}$.<smiles>[R]c1ccc(C(=O)Nc2nc3cc(Cl)ccc3s2)cc1[R]</smiles>

3<smiles>[R]c1ccc2nc(N)sc2c1</smiles>

5

\begin{tabular}{lcc} 
Compound & $\mathrm{R}$ & $\mathrm{R}^{1}$ \\
\hline $\mathbf{3 a}$ & $4-\mathrm{F}$ & $2,3-\mathrm{F}_{2}$ \\
$\mathbf{3 b}$ & $4-\mathrm{F}$ & $2,4-\mathrm{F}_{2}$ \\
$\mathbf{3 c}$ & $4-\mathrm{F}$ & $2,5-\mathrm{F}_{2}$ \\
$\mathbf{3 d}$ & $4-\mathrm{F}$ & $2,6-\mathrm{F}_{2}$ \\
$\mathbf{3 e}$ & $4-\mathrm{F}$ & $3,4-\mathrm{F}_{2}$ \\
$\mathbf{3 f}$ & $4-\mathrm{F}$ & $3,5-\mathrm{F}_{2}$ \\
$\mathbf{3 g}$ & $5-\mathrm{F}$ & $2,3-\mathrm{F}_{2}$ \\
$\mathbf{3 h}$ & $5-\mathrm{F}$ & $2,4-\mathrm{F}_{2}$ \\
$\mathbf{3 i}$ & $5-\mathrm{F}$ & $2,5-\mathrm{F}_{2}$ \\
$\mathbf{3 j}$ & $5-\mathrm{F}$ & $2,6-\mathrm{F}_{2}$ \\
$\mathbf{3 k}$ & $5-\mathrm{F}$ & $3,4-\mathrm{F}_{2}$ \\
$\mathbf{3 1}$ & $5-\mathrm{F}$ & $3,5-\mathrm{F}_{2}$ \\
$\mathbf{5 a}$ & $4-\mathrm{F}$ & - \\
$\mathbf{5 b}$ & $5-\mathrm{F}$ & - \\
\hline
\end{tabular}

indications about the effects of halogen substitutions on several amides of 1,3-benzothiazole previously reported ( $N$ 1,3-benzothiazol-2-ylbenzamides, 1, Figure 1). We found that these compounds exerted antifungal activity against C. albicans comparable to that of the reference compound sorbic acid, that is, with MIC values in the order of $250-500 \mu \mathrm{g} / \mathrm{mL}$ [34]. Nowadays, it is known that sorbic acid is no longer the best reference compound to be used to study antifungal activity; it is preferable to use antibiotics, such as amphotericin or fluconazole, which are much more active showing MIC values in the order of $0.5-1 \mu \mathrm{g} / \mathrm{mL}$; thus, the compounds previously reported can be considered inactive against fungi. In this paper, we report a series of trifluoro-substituted$N$-(6-chloro-1,3-benzothiazol-2-yl) benzamides (3, Table 1), that are position isomers of those previously reported [34]. Moreover, 2-amino-1,3-benzothiazoles (5, Table 1) were also synthesized and tested, and the results were compared to those obtained with their isomers previously reported (2, Figure 1) [35].

\section{Results and Discussion}

Compounds $\mathbf{5 a}, \mathbf{b}$ and $\mathbf{3} \mathbf{a}-\mathbf{l}$ were prepared as reported in Scheme 1 . The appropriate fluoro-4-chloro aniline $(\mathbf{4 a}, \mathbf{b})$ was reacted with bromine and potassium thiocyanate to give the corresponding 2 -amino benzothiazoles $\mathbf{5 a}, \mathbf{b}$, which were reacted with the suitable difluorobenzoyl chloride to give all possible isomeric compounds $3 \mathbf{a}-\mathbf{l}$. The antimicrobial activity of compounds 3a-l was evaluated in vitro against Grampositive and Gram-negative bacteria (Staphylococcus aureus 29213, and Enterococcus faecalis 29212, Escherichia coli 25922) and fungi strains (Candida albicans 10231, Candida parapsilosis 22019, and Candida tropicalis 750) belonging to the ATCC collection [36, 37]. All MIC determinations were carried out according to the clinical laboratory standards institute (CLSI) guidelines. MIC values are given in $\mu \mathrm{g} / \mathrm{mL}$ and were compared to MIC values for the standard antibacterial drugs oxacillin and norfloxacin and antifungal fluconazole. Screening results are summarized in Table 2 . The combined data showed that seven compounds, out of twelve, exerted interesting inhibitory activity against $E$. faecalis with MIC values between 8 and $32 \mu \mathrm{g} / \mathrm{mL}$. In particular, compound $3 \mathbf{i}$ was the most active derivative giving the best antibacterial activity against E. faecalis with an MIC value of $8 \mu \mathrm{g} / \mathrm{mL}$, while compounds $\mathbf{3 a}, \mathbf{b}, \mathbf{f}-\mathbf{h}$, and $\mathbf{k}$ displayed moderate activity towards the same bacteria strain (MIC: $32 \mu \mathrm{g} / \mathrm{mL}$ ). In order to better define structure activity relationships, it is possible to consider two subseries of compounds: the former, 6chloro-4-fluorobenzothiazole benzamides (3a-f); the latter, 6-chloro-5-fluorobenzothiazole benzamides (3g-1). Among all the tested compounds, with the exception of the highly active compound $\mathbf{3 i}$, the best substitutions of the acyl moiety seem to be 2,3-difluoro and 2,4-difluoro in both series (3a, b and $\mathbf{3 g}, \mathbf{h}$ ) and the worst substitution seems to be 2,6-difluoro in both series (3d and $\mathbf{3 j}$ ). Moreover, the 3,4-difluoro and 3,5difluoro substitutions had different effects in the two series: in particular, the former decreases activity in the first series (3e), while it increased activity in the second (3k), and the opposite was true for the latter substitution ( $3 \mathbf{f}$ was active, and 31 was inactive). Then, 2,5-difluorosubstitution determined a very high increase in activity in the second series giving the best compound tested (3i) while decreasing the activity in the first series (3c; MIC: $128 \mu \mathrm{g} / \mathrm{mL}$ ). Finally, 2,6-difluoro substitution was detrimental in both series: compounds $\mathbf{3 d}$ and $3 \mathbf{j}$ showed no activity or low activity against $E$. faecalis (MIC > $512 \mu \mathrm{g} / \mathrm{mL}$ and MIC: $128 \mu \mathrm{g} / \mathrm{mL}$, resp.). All the compounds did show very low or no activity against $S$. aureus and $E$. coli and all the strains of Candida. These results are in agreement with what had been found for a series of isomers of our compounds previously reported [34]. Moreover, intermediates $\mathbf{5 a}, \mathbf{b}$ were tested in order to compare results obtained with their isomers recently reported, in which the halogen atoms were inverted in their position [35]. These 




Scheme 1: Reagents and conditions: (i) $\mathrm{Br}_{2}, \mathrm{KSCN}, \mathrm{AcOH}, 30-35^{\circ} \mathrm{C}$; (ii) suitable difluorobenzoyl chloride, $\mathrm{Et}_{3} \mathrm{~N}, \mathrm{dioxane} 50-60^{\circ} \mathrm{C}$.

TABLE 2: Antimicrobial activity results of benzothiazole derivatives $\mathbf{3 a - 1}$ and $\mathbf{5 a}, \mathbf{b}(\mathrm{MIC}, \mu \mathrm{g} / \mathrm{mL})$.

\begin{tabular}{|c|c|c|c|c|c|c|}
\hline & & & roorganism (MIC & & & \\
\hline & & & Gram negative $\mathrm{e}^{\mathrm{a}}$ & & Fungi $^{\mathrm{b}}$ & \\
\hline & S.a. 29213 & E.f. 29212 & E.c. 25922 & c.a. 10231 & C.p. 22019 & C.t. 750 \\
\hline $3 a$ & 64 & 32 & $\mathrm{R}$ & $\mathrm{R}$ & $\mathrm{R}$ & $\mathrm{R}$ \\
\hline $3 b$ & $\mathrm{R}$ & 32 & $\mathrm{R}$ & $\mathrm{R}$ & $\mathrm{R}$ & $\mathrm{R}$ \\
\hline $3 c$ & 256 & 256 & $\mathrm{R}$ & $\mathrm{R}$ & $\mathrm{R}$ & $\mathrm{R}$ \\
\hline $3 d$ & $\mathrm{R}$ & $\mathrm{R}$ & 256 & $\mathrm{R}$ & $\mathrm{R}$ & $\mathrm{R}$ \\
\hline $3 e$ & $\mathrm{R}$ & $\mathrm{R}$ & $\mathrm{R}$ & $\mathrm{R}$ & $\mathrm{R}$ & $\mathrm{R}$ \\
\hline $3 f$ & $\mathrm{R}$ & 32 & $\mathrm{R}$ & $\mathrm{R}$ & $\mathrm{R}$ & $\mathrm{R}$ \\
\hline $3 g$ & $\mathrm{R}$ & 32 & $\mathrm{R}$ & $\mathrm{R}$ & $\mathrm{R}$ & $\mathrm{R}$ \\
\hline $3 \mathrm{~h}$ & $\mathrm{R}$ & 32 & $\mathrm{R}$ & $\mathrm{R}$ & $\mathrm{R}$ & $\mathrm{R}$ \\
\hline $3 \mathbf{i}$ & 128 & 8 & $\mathrm{R}$ & $\mathrm{R}$ & $\mathrm{R}$ & $\mathrm{R}$ \\
\hline $3 \mathbf{j}$ & 256 & 128 & $\mathrm{R}$ & $\mathrm{R}$ & $\mathrm{R}$ & $\mathrm{R}$ \\
\hline $3 k$ & $\mathrm{R}$ & 32 & 256 & $\mathrm{R}$ & $\mathrm{R}$ & $\mathrm{R}$ \\
\hline 31 & $\mathrm{R}$ & $\mathrm{R}$ & $\mathrm{R}$ & $\mathrm{R}$ & $\mathrm{R}$ & $\mathrm{R}$ \\
\hline $5 a$ & 256 & 256 & $\mathrm{R}$ & 128 & 64 & 128 \\
\hline $5 b$ & 128 & 128 & 256 & 64 & 128 & 128 \\
\hline OXA & 0.25 & 16 & $\mathrm{R}$ & - & - & - \\
\hline NRF & 0.5 & 4 & 0.03 & - & - & - \\
\hline FCN & - & - & - & 2 & 2 & 4 \\
\hline
\end{tabular}

${ }^{a}$ Antibacterial activity was estimated by using CLSI assay [36]. Abbreviations: S.a.: S. aureus; E.f:: E. faecalis; E.c.: E. coli; OXA: oxacillin; NRF: norfloxacin; R: resistant $(>512 \mu \mathrm{g} / \mathrm{mL})$.

${ }^{\mathrm{b}}$ Antifungal activity was estimated by using CLSI assay [37]. Abbreviations: C.a.: C. albicans; C.p.: C. parapsilosis; C.t.: C. tropicalis; C.k.: C. krusei; FCN: fluconazole; R: resistant ( $>512 \mu \mathrm{g} / \mathrm{mL})$.

two compounds did show no antimicrobial activity, while results found as antifungals are similar to their corresponding isomers. In particular, compound 5a was identical to its isomer against Gram-positive and -negative bacteria and against $C$. parapsilosis, while it was slightly more active than their isomers previously reported [35] on C. albicans and $C$. tropicalis (MIC: $128 \mu \mathrm{g} / \mathrm{mL}$ versus $256 \mu \mathrm{g} / \mathrm{mL}$ ). Compound 5b was identical to its isomer against Gram-negative and C. tropicalis, slightly more active against $S$. aureus (MIC: $128 \mu \mathrm{g} / \mathrm{mL}$ versus $512 \mu \mathrm{g} / \mathrm{mL}$ ) and E. faecalis (MIC: $128 \mu \mathrm{g} / \mathrm{mL}$ versus $256 \mu \mathrm{g} / \mathrm{mL}$ ) and C. albicans (MIC: $64 \mu \mathrm{g} / \mathrm{mL}$ versus $128 \mu \mathrm{g} / \mathrm{mL})$, and slightly less active against C. parapsilosis (MIC: $128 \mu \mathrm{g} / \mathrm{mL}$ versus $64 \mu \mathrm{g} / \mathrm{mL}$ ).

\section{Conclusion}

In conclusion, we report the synthesis and antimicrobial activity of a series of 1,3-benzothiazole derivatives (3a-1 and $5 \mathbf{a}, \mathbf{b})$. All the compounds did not show activity against $S$. aureus, E. coli and C. albicans, C. parapsilosis, and C. tropicalis in these tracing results previously obtained for the corresponding position isomers previously reported $(\mathbf{1}, \mathbf{2}$, Figure 1$)$ $[34,35]$. Interestingly, compounds $\mathbf{3 a}, \mathbf{b}, \mathbf{f}, \mathbf{g}, \mathbf{h}, \mathbf{i}$, and $\mathbf{k}$ exerted moderate to high activity against E. faecalis (MIC between 8 and $32 \mu \mathrm{g} / \mathrm{mL}$ ). In particular, compound $3 \mathbf{i}$ was the most potent of the series (MIC: $8 \mu \mathrm{g} / \mathrm{mL}$ ) and the most promising compound, while the other showed an MIC value of $32 \mu \mathrm{g} / \mathrm{mL}$.

\section{Experimental}

4.1. General Experimental Details. Chemicals were purchased from Sigma-Aldrich or Lancaster. Yields refer to purified products and were not optimized. The structures of the compounds were confirmed by routine spectrometric and spectroscopic analyses. Only spectra for compounds not previously described are given. Purity of compounds was assessed by GC analysis. Melting points were determined on a Gallenkamp apparatus in open glass capillary tubes and are uncorrected. Infrared spectra were recorded 
on a Perkin-Elmer (Norwalk, CT) Spectrum One FT spectrophotometer, and band positions are given in reciprocal centimeters $\mathrm{cm}^{-1}$. ${ }^{1} \mathrm{H}$ NMR spectra were recorded on a Varian VX Mercury spectrometer operating at $300 \mathrm{MHz}$ using $\mathrm{CDCl}_{3}$ and DMSO- $d_{6}$ as solvents. Chemical shifts are reported in parts per million (ppm) relative to the residual nondeuterated solvent resonance: $\mathrm{CDCl}_{3}, \delta 7.26$ and DMSO- $d_{6}, \delta$ 2.48. $J$ values are given in Hz. GC-MS was performed on a Hewlett-Packard 6890-5973 MSD at low resolution. Chromatographic separations were performed on silica gel columns by flash chromatography (Kieselgel 60, 0.040-0.063 mm, Merck, Darmstadt, Germany) as previously reported [38-41]. TLC analyses were performed on precoated silica gel on aluminum sheets (Kieselgel $60 \mathrm{~F}_{254}$, Merck). GC analyses were performed on a Varian 3800 gas chromatograph equipped with a flame ionization detector and a Jew Scientific DB- 5 capillary column (30 m length $\times 0.25 \mathrm{~mm}$ ID, $0.25 \mu \mathrm{m}$ film thickness) [42].

4.1.1. N-(6-Chloro-4-fluoro-1,3-benzothiazol-2-yl)-2,3 difluorobenzamide (3a). A mixture of $5 \mathbf{a}(0.61 \mathrm{~g}, 3.0 \mathrm{mmol})$ and triethylamine $(0.30 \mathrm{~g}, 3.0 \mathrm{mmol})$ in dry dioxane $(30 \mathrm{~mL})$ was stirred for $30 \mathrm{~min}$ at $50-60^{\circ} \mathrm{C}$. A solution of 2,3-difluorobenzoyl chloride $(0.53 \mathrm{~g}, 3.0 \mathrm{mmol})$ in dry dioxane $(30 \mathrm{~mL})$ was added dropwise. The mixture was stirred for $2 \mathrm{~h}$ and then poured into crushed ice. The resulting solid, so separated, was collected by filtration and washed with $1 \%$ potassium bicarbonate aqueous solution. The crude residue was purified by column chromatography on silica gel (EtOAc/petroleum ether $3: 7)$ to give $0.32 \mathrm{~g}(31 \%)$ of $3 \mathbf{a}$ as a white solid: $\mathrm{mp} 240-$ $242^{\circ} \mathrm{C}$; GC-MS (70 eV, electron impact) $\mathrm{m} / z(\%) 342\left(\mathrm{M}^{+}, 38\right)$, 141 (100); ${ }^{1} \mathrm{H}$ NMR: $\delta$ 7.18-7.25 (m, 1H, Ar HC-5), 7.26-7.35 (m, 1H, Ar HC-3 ${ }^{\prime}$ ), 7.42-7.55 (m, 1H, Ar HC-4' $), 7.63$ (s, 1H, Ar $\left.H C-5^{\prime}\right), 7.96$ (t, $J=6.3 \mathrm{~Hz}, 1 \mathrm{H}$, Ar HC-7), 10.08 (br s, $1 \mathrm{H}$, $\mathrm{NH}$, exch $\left.\mathrm{D}_{2} \mathrm{O}\right)$; IR (KBr): $3405(\mathrm{NH}), 1686(\mathrm{C}=\mathrm{O}) \mathrm{cm}^{-1}$.

4.1.2. N-(6-Chloro-4-fluoro-1,3-benzothiazol-2-yl)-2,4-difluorobenzamide $(\mathbf{3} \boldsymbol{b})$. Prepared as reported previously for $\mathbf{3 a}$ starting from $\mathbf{5 a}$ and 2,4-difluorobenzoyl chloride. Yield: $33 \%$; white solid: $\mathrm{mp}>250^{\circ} \mathrm{C}$; GC-MS (70 eV, electron impact) $m / z$ (\%) $342\left(\mathrm{M}^{+}, 18\right), 141$ (100); ${ }^{1} \mathrm{H}$ NMR (DMSO$\left.d_{6}\right): \delta$ 7.12-7.55 (m, 3H, Ar HC-5 + Ar HC-3' $\left.5^{\prime}\right), 7.85-$ 8.05 ppm (m, 2H, Ar HC-7 +Ar HC-6' ${ }^{\prime}$; IR (KBr): $3418(\mathrm{NH})$, $1673(\mathrm{C}=\mathrm{O}) \mathrm{cm}^{-1}$.

4.1.3. N-(6-Chloro-4-fluoro-1,3-benzothiazol-2-yl)-2,5-difluorobenzamide $(3 \boldsymbol{c})$. Prepared as reported previously for $3 \mathbf{a}$ starting from 5a and 2,5-difluorobenzoyl chloride. Yield: 24\%; white solid: $\mathrm{mp}>250^{\circ} \mathrm{C}$; GC-MS (70 eV, electron impact) $m / z(\%) 342\left(\mathrm{M}^{+}, 17\right), 141(100) ;{ }^{1} \mathrm{H}$ NMR: $\delta 7.00-$ 7.25 (m, 2H, Ar HC-5 + HC-4' ${ }^{\prime}$, 7.50-7.65 (m, 1H, Ar HC- $6^{\prime}$ ), 7.70-7.85 (m, 1H, Ar HC-3'), 8.02 (d, J=5.2 Hz, 1H, Ar HC-7), 10.22 ppm (br s, $1 \mathrm{H}, \mathrm{NH})$; IR (KBr): $3410(\mathrm{NH}), 1677(\mathrm{C}=\mathrm{O})$ $\mathrm{cm}^{-1}$.

4.1.4. N-(6-Chloro-4-fluoro-1,3-benzothiazol-2-yl)-2,6-difluorobenzamide (3d). Prepared as reported previously for $\mathbf{3 a}$ starting from 5a and 2,6-difluorobenzoyl chloride. Yield: 12\%; white solid: mp $245-247^{\circ} \mathrm{C}$; GC-MS (70 eV, electron impact) $m / z(\%) 342\left(\mathrm{M}^{+}, 18\right), 141(100) ;{ }^{1} \mathrm{H}$ NMR: $\delta 7.01(\mathrm{t}, J=8.5 \mathrm{~Hz}$, $\left.2 \mathrm{H}, \mathrm{Ar} \mathrm{HC}-3^{\prime}, 5^{\prime}\right), 7.14$ (dd, $J=10.1,1.8 \mathrm{~Hz}, 1 \mathrm{H}$, Ar HC-5), 7.447.58 (m, 1H, Ar, HC-4' $), 7.63$ (s, 1H Ar HC-7), 10.37 ppm (br s, $1 \mathrm{H}, \mathrm{NH})$; IR (KBr): $3409(\mathrm{NH}), 1694(\mathrm{C}=\mathrm{O}) \mathrm{cm}^{-1}$.

4.1.5. N-(6-Chloro-4-fluoro-1,3-benzothiazol-2-yl)-3,4-difluorobenzamide $(\mathbf{3 e})$. Prepared as reported previously for $\mathbf{3 a}$ starting from $\mathbf{5 a}$ and 3,4-difluorobenzoyl chloride. Yield: $14 \%$; slightly yellowish solid: mp $226-228^{\circ} \mathrm{C}$; GC-MS $(70 \mathrm{eV}$, electron impact) $m / z(\%) 342\left(\mathrm{M}^{+}, 26\right), 141(100) ;{ }^{1} \mathrm{H}$ NMR: $\delta 7.19$ (dd, $J=9.9,1.6 \mathrm{~Hz}, 1 \mathrm{H}$, Ar HC-5), 7.25-7.40 (m, 1H, Ar HC- $5^{\prime}$ ), 7.62 (s, 1H Ar HC-7), 7.80-7.88 (m, 1H, Ar HC-2' $)$, 7.90-8.02 ppm (m, 1H, Ar HC-6' ); IR (KBr): 3410 (NH), 1679 $(\mathrm{C}=\mathrm{O}) \mathrm{cm}^{-1}$.

4.1.6. N-(6-Chloro-4-fluoro-1,3-benzothiazol-2-yl)-3,5-difluorobenzamide (3f). Prepared as reported previously for $\mathbf{3 a}$ starting from 5a and 3,5-difluorobenzoyl chloride. Yield: $34 \%$; white solid: $\mathrm{mp}>250^{\circ} \mathrm{C}$; GC-MS $(70 \mathrm{eV}$, electron impact) $m / z(\%) 342\left(\mathrm{M}^{+}, 36\right), 141(100) ;{ }^{1} \mathrm{H}$ NMR: $\delta 7.10(\mathrm{tt}$, $\left.J=8.2,2.2 \mathrm{~Hz}, 1 \mathrm{H}, \mathrm{Ar} H \mathrm{C}-4^{\prime}\right), 7.21(\mathrm{dd}, J=9.9,1.9 \mathrm{~Hz}, 1 \mathrm{H}, \mathrm{Ar}$ HC-5), 7.47-7.57 (m, 2H, Ar HC-2' $\left.6^{\prime}\right), 7.62-7.66 \mathrm{ppm}(\mathrm{m}, 1 \mathrm{H}$, Ar HC-7); IR (KBr): $3414(\mathrm{NH}), 1681(\mathrm{C}=\mathrm{O}) \mathrm{cm}^{-1}$.

4.1.7. N-(6-Chloro-5-fluoro-1,3-benzothiazol-2-yl)-2,3-difluorobenzamide (3g). Prepared as reported previously for $\mathbf{3 a}$ starting from $\mathbf{5 b}$ and 2,3-difluorobenzoyl chloride. Yield: 64\%; white solid: mp 241-243 ${ }^{\circ} \mathrm{C}$; GC-MS (70 eV, electron impact) $m / z(\%) 342\left(\mathrm{M}^{+}, 25\right), 141(100) ;{ }^{1} \mathrm{H}$ NMR: $\delta 7.25-$ 7.38 (m, 1H, Ar HC-4' ${ }^{\prime}$ ), 7.42-7.52 (m, 1H, Ar HC-5' ), 7.59 (d, $J=9.3 \mathrm{~Hz}, 1 \mathrm{H}$, Ar HC-4), $7.86(\mathrm{~d}, J=7.1 \mathrm{~Hz}, 1 \mathrm{H}$, Ar HC-7), 7.93-8.02 ppm (m, 1H, Ar HC-6' $)$; IR (KBr): 3414 (NH), 1673 $(\mathrm{C}=\mathrm{O}) \mathrm{cm}^{-1}$.

4.1.8. N-(6-Chloro-5-fluoro-1,3-benzothiazol-2-yl)-2,4-difluorobenzamide (3h). Prepared as reported previously for $\mathbf{3 a}$ starting from $\mathbf{5 b}$ and 2,4-difluorobenzoyl chloride. Yield: $64 \%$; beige solid, $\mathrm{mp}>250^{\circ} \mathrm{C}$; GC-MS $(70 \mathrm{eV}$, electron impact) $m / z(\%) 342\left(\mathrm{M}^{+}, 17\right), 141$ (100); ${ }^{1} \mathrm{H}$ NMR (DMSO$\left.d_{6}\right): \delta 7.16-7.32(\mathrm{~m}, 1 \mathrm{H}$, Ar HC-3') $7.40-7.56(\mathrm{~m}, 1 \mathrm{H}, \mathrm{Ar} H \mathrm{C}-$ $5^{\prime}$ ), 7.80-7.96 (m overlapping d at $7.84 \mathrm{ppm}, 1 \mathrm{H}, \mathrm{Ar} H \mathrm{C}-6^{\prime}$ ), 7.84 (d overlapping $\mathrm{m}$ at $7.80-7.96 \mathrm{ppm}, J=10.2 \mathrm{~Hz}, 1 \mathrm{H}, \mathrm{Ar}$ HC-4), 8.32 (d, $J=7.7 \mathrm{~Hz}, 1 \mathrm{H}$, Ar HC-7), 13.04 ppm (br s, $1 \mathrm{H}$, $\mathrm{NH})$; IR (KBr): $3420(\mathrm{NH}), 1671(\mathrm{C}=\mathrm{O}) \mathrm{cm}^{-1}$.

4.1.9. N-(6-Chloro-5-fluoro-1,3-benzothiazol-2-yl)-2,5-difluorobenzamide (3i). Prepared as reported previously for $\mathbf{3 a}$ starting from $\mathbf{5 b}$ and 2,5-difluorobenzoyl chloride. Yield: 64\%; white solid, $\mathrm{mp}>250^{\circ} \mathrm{C}$; GC-MS (70 eV, electron impact) $m / z(\%) 342\left(\mathrm{M}^{+}, 15\right), 141(100) ;{ }^{1} \mathrm{H}$ NMR: $\delta 7.15-7.40$ (m, 2H, Ar HC-3' $5^{\prime}$ ), 7.59 (d, $J=9.3 \mathrm{~Hz}, 1 \mathrm{H}$, Ar HC-4), 7.87 (d, $J=6.9 \mathrm{~Hz}, 1 \mathrm{H}$, Ar HC-7), 7.90-7.98 (m, 1H, Ar HC-6' $)$, 10.05 ppm (br s, 1H, NH); IR (KBr): $3496(\mathrm{NH}), 1682(\mathrm{C}=\mathrm{O})$ $\mathrm{cm}^{-1}$.

4.1.10. N-(6-Chloro-5-fluoro-1,3-benzothiazol-2-yl)-2,6-difluorobenzamide (3j). Prepared as reported previously for $\mathbf{3 a}$ starting from $\mathbf{5 b}$ and 2,6-difluorobenzoyl chloride. Yield: 70\%; white solid, mp 247-249 ${ }^{\circ}$; GC-MS (70 eV, electron 
impact) $m / z(\%) 342\left(\mathrm{M}^{+}, 18\right), 141(100) ;{ }^{1} \mathrm{H}$ NMR: $\delta 7.03(\mathrm{t}, J$ $=9.0 \mathrm{~Hz}, 1 \mathrm{H}$, Ar HC- $\left.3^{\prime}, 5^{\prime}\right), 7.34(\mathrm{~d}, J=9.3 \mathrm{~Hz}, 1 \mathrm{H}$, Ar HC-4), 7.45-7.58 (m, 1H, Ar HC-4' $), 7.85$ ppm $(\mathrm{d}, J=6.9 \mathrm{~Hz}, 1 \mathrm{H}, \mathrm{Ar}$ HC-7); IR (KBr): $3430(\mathrm{NH}), 1687(\mathrm{C}=\mathrm{O}) \mathrm{cm}^{-1}$.

4.1.11. N-(6-Chloro-5-fluoro-1,3-benzothiazol-2-yl)-3,4-difluorobenzamide (3k). Prepared as reported previously for $\mathbf{3 a}$ starting from $\mathbf{5 b}$ and 3,4-difluorobenzoyl chloride. Yield: $62 \%$; white solid, $\mathrm{mp}>250^{\circ} \mathrm{C}$; GC-MS $(70 \mathrm{eV}$, electron impact) $m / z(\%) 342\left(\mathrm{M}^{+}, 33\right), 141(100) ;{ }^{1} \mathrm{H}$ NMR: $\delta$ 7.25-7.40 (m, 1H, Ar HC-5), 7.48 (d, $J=9.3 \mathrm{~Hz}, 1 \mathrm{H}$, Ar HC-4), 7.767.85 (m, 1H, Ar HC-2' $), 7.86$ (d, $J=6.9 \mathrm{~Hz}, 1 \mathrm{H}$, Ar HC-7), 7.87-7.98 ppm (m, 1H, Ar HC-6' ); IR (KBr): 3408 (NH), 1682 $(\mathrm{C}=\mathrm{O}) \mathrm{cm}^{-1}$.

4.1.12. N-(6-Chloro-5-fluoro-1,3-benzothiazol-2-yl)-3,5-difluorobenzamide (3l). Prepared as reported previously for $\mathbf{3 a}$ starting from $\mathbf{5 b}$ and 3,5-difluorobenzoyl chloride. Yield: $55 \%$; white solid, mp 235-237 ${ }^{\circ}$; GC-MS (70 eV, electron impact) $m / z(\%) 342\left(\mathrm{M}^{+}, 39\right), 141(100) ;{ }^{1} \mathrm{H}$ NMR (DMSO$\left.d_{6}\right): \delta 7.55-7.65\left(\mathrm{~m}, 1 \mathrm{H}, \mathrm{Ar} H \mathrm{HC}-4^{\prime}\right), 7.70-7.90(\mathrm{~m}, 3 \mathrm{H}, \mathrm{HC}-4,-$ $\left.2^{\prime}, 6^{\prime}\right), 8.33$ (d, $J=7.4 \mathrm{~Hz}, 1 \mathrm{H}$, Ar HC-7), $13.2 \mathrm{ppm}$ (br s, $1 \mathrm{H}$, $\mathrm{NH})$; IR (KBr): $3401(\mathrm{NH}), 1679(\mathrm{C}=\mathrm{O}) \mathrm{cm}^{-1}$.

4.1.13. 6-Chloro-4-fluoro-1,3-benzothiazol-2-amine (5a). A mixture of $4 \mathbf{a}(15 \mathrm{~g}, 103 \mathrm{mmol})$ and potassium thiocyanate $(20 \mathrm{~g}, 206 \mathrm{mmol})$ in glacial acetic acid $(250 \mathrm{~mL})$ was stirred for $5 \mathrm{~min}$. Bromine $(24 \mathrm{~g}, 150 \mathrm{mmol})$ in glacial acetic acid $(250 \mathrm{~mL})$ was added dropwise to this mixture, with the temperature being kept below $30-35^{\circ} \mathrm{C}$ throughout the addition. Stirring was continued for an additional $1 \mathrm{~h}$ after addition of bromine. After cooling, the residue was removed by filtration. The filtered solution was made alkaline with $28 \%$ ammonium hydroxide, and the solid precipitate was collected and washed with water. The combined water layers were made alkaline with $28 \%$ ammonium hydroxide, and the resulting precipitate was combined with that previously collected. The combined precipitates were extracted with EtOAc. The organic layers were separated, dried $\left(\mathrm{Na}_{2} \mathrm{SO}_{4}\right)$, and evaporated under vacuum. The crude residue was purified by column chromatography on silica gel (hexane/EtOAc $3: 7$ ) to give $14.6 \mathrm{~g}(70 \%)$ of $\mathbf{5 a}$ as a slight green solid: $\mathrm{mp} 243-245^{\circ} \mathrm{C}$; GC-MS (70 eV, electron impact) $\mathrm{m} / z$ (\%) $202\left(\mathrm{M}^{+}, 100\right) ;{ }^{1} \mathrm{H}$ NMR: $\delta 7.08$ (dd, $J=10.2,1.9 \mathrm{~Hz}, 1 \mathrm{H}, \mathrm{Ar} H \mathrm{C}-7), 7.30-7.40(\mathrm{~m}$, $1 \mathrm{H}, H \mathrm{C}-5), 5.63 \mathrm{ppm}$ (br s, $1 \mathrm{H}, \mathrm{NH}_{2}$ ); IR (KBr): 3461, 3074 $\left(\mathrm{NH}_{2}\right) \mathrm{cm}^{-1}$.

4.1.14. 6-Chloro-5-fluoro-1,3-benzothiazol-2-amine (5b). Prepared as reported previously for $\mathbf{5} \mathbf{a}$ starting from $\mathbf{4 b}$. Yield: 65\%; white solid: mp 234-236 C; GC-MS (70 eV, electron impact) $m / z(\%) 202\left(\mathrm{M}^{+}, 100\right){ }^{1} \mathrm{H} \mathrm{NMR}: \delta 7.30(\mathrm{~d}, J=9.9 \mathrm{~Hz}$, $1 \mathrm{H}, \mathrm{Ar} H \mathrm{C}-4), 7.56$ (d, $J=6.9 \mathrm{~Hz}, 1 \mathrm{H}$, Ar HC-7), $5.45 \mathrm{ppm}$ (br s, $\left.1 \mathrm{H}, \mathrm{NH}_{2}\right)$; IR (KBr): 3473, $3083\left(\mathrm{NH}_{2}\right) \mathrm{cm}^{-1}$.

\subsection{Biology}

4.2.1. Antibacterial Studies. The in vitro minimum inhibitory concentrations (MICs, $\mu \mathrm{g} / \mathrm{mL}$ ) were assessed by the broth microdilution method, using 96-well plates, according to CLSI guidelines [36]. Stock solutions of the tested compounds were obtained in DMSO. Stock solutions of lower concentrations were prepared for those substances which did not dissolve well. Then twofold serial dilutions in the suitable test medium between 512 and $0.5 \mu \mathrm{g} / \mathrm{mL}$ were plated. To be sure that the solvent had no adverse effect on bacterial growth, a control test was carried out by using DMSO at its maximum concentration along with the medium. Bacteria strains available as freeze-dried discs, belonging to the ATCC collection, were used: Gram-positive strains such as Staphylococcus aureus 29213 and Enterococcus faecalis 29212 and Gram-negative one such as Escherichia coli 25922. To preserve the purity of cultures and to allow the reproducibility, a series of cryovials of all microbial strains in $10 \%$ glycerol medium was set up and stored at $-80^{\circ} \mathrm{C}$. Precultures of each bacterial strain were prepared in cation-adjusted mueller-hinton broth (CAMHB) and incubated at $37^{\circ} \mathrm{C}$ until the growth ceased. The turbidity of bacterial cell suspension was calibrated to $0.5 \mathrm{McF}$ arland Standard by spectrophotometric method $(625 \mathrm{~nm}$, range $0.08-0.10)$, and further the standardized suspension was diluted 1:100 with CAMHB to have 1-2 $\times$ $10^{6} \mathrm{CFU} / \mathrm{mL}$. All wells were seeded with $100 \mu \mathrm{L}$ of inoculum. A number of wells containing only inoculated broth as control growth were prepared. The plates were incubated at $37^{\circ} \mathrm{C}$ for $24 \mathrm{~h}$, and the MIC values were recorded as the last well containing no bacterial growth. The MICs were determined by using an antibacterial assayed repeated twice in triplicate. Oxacillin and norfloxacin were used as reference drugs.

4.2.2. Antifungal Studies. Antifungal studies [37] were carried out against Candida albicans 10231, Candida parapsilosis 22019, Candida tropicalis 750, and Candida krusei 6258, belonging to the ATCC collection. Preparation of stock solutions and purity of cultures preservation were obtained as previously described for antibacterial studies. Pre-cultures of each yeast strain were prepared in Sabouraud broth (SAB) $2 \%$ glucose, and incubated at $37^{\circ} \mathrm{C}$ until the growth ceased. The turbidity of yeast stock suspension was calibrated to $0.5 \mathrm{McF}$ arland Standard by spectrophotometric method $(530 \mathrm{~nm}$, range $0.12-0.15)$, and further the standardized suspension was diluted first $1: 50$ with SAB and then $1: 20$ in the same medium to have $1-5 \times 10^{6} \mathrm{CFU} / \mathrm{mL}$. All wells were seeded with $100 \mu \mathrm{L}$ of inoculum. A number of wells containing only inoculated broth as control growth were prepared. The plates were incubated at $37^{\circ} \mathrm{C}$ for $24-48 \mathrm{~h}$, and the MIC values were recorded as the last well containing no fungal growth. The MICs were determined by using an antifungal assay repeated twice in triplicates. Fluconazole was used as a reference drug.

\section{Acknowledgment}

This work was accomplished thanks to the financial support of the Ministero dell'Istruzione, dell'Università e della Ricerca (MIUR). 


\section{References}

[1] S. DebRoy, R. van der Hoeven, K. V. Singh et al., "Development of a genomic site for gene integration and expression in Enterococcus faecalis," Journal of Microbiological Methods, vol. 90, no. 1, pp. 1-8, 2012.

[2] T. Yasufuku, K. Shigemura, T. Shirakawa et al., "Mechanisms of and risk factors for fluoroquinolone resistance in clinical Enterococcus faecalis isolates from patients with urinary tract infections," Journal of Clinical Microbiology, vol. 49, no. 11, pp. 39123916, 2011.

[3] N. Conceição, C. da Cunha Hueb Barata de Oliveira, P. R. da Silva, B. G. M. Ávila, and A. G. de Oliveira, "Trends in antimicrobial resistance among clinical isolates of enterococci in a Brazilian tertiary hospital: a 4-year study," Revista da Sociedade Brasileira de Medicina Tropical, vol. 44, no. 2, pp. 177-181, 2011.

[4] C. Pozzi, S. Ferrari, D. Cortesi et al., "The X-ray crystal structure of Enterococcus faecalis thymidylate synthase provides clues about folate bacterial metabolism," Acta Crystallographica D, vol. 68, no. 9, pp. 1232-1241, 2012.

[5] K. Ishikawa, T. Matsumoto, M. Yasuda et al., "The nationwide study of bacterial pathogens associated with urinary tract infections conducted by the Japanese Society of Chemotherapy," Journal of Infection and Chemotherapy, vol. 17, no. 1, pp. 126-138, 2011.

[6] D. A. Horton, G. T. Bourne, and M. L. Smythe, "The combinatorial synthesis of bicyclic privileged structures or privileged substructures," Chemical Reviews, vol. 103, no. 3, pp. 893-930, 2003.

[7] L. A. Thompson and J. A. Ellman, "Synthesis and applications of small molecule libraries," Chemical Reviews, vol. 96, no. 1, pp. 555-600, 1996.

[8] J. S. Früchtel and G. Jung, "Organic chemistry on solid supports," Angewandte Chemie, vol. 35, no. 1, pp. 17-42, 1996.

[9] A. Nefzi, J. M. Ostresh, and R. A. Houghten, "The current status of heterocyclic combinatorial libraries," Chemical Reviews, vol. 97, no. 2, pp. 449-472, 1997.

[10] R. G. Franzén, "Recent advances in the preparation of heterocycles on solid support: a review of the literature," Journal of Combinatorial Chemistry, vol. 2, no. 3, pp. 195-214, 2000.

[11] M. Kamal, A. K. Shakya, and T. Jawaid, "1, 3, 4-Thiadiazole as antimicrobial agent: a review," International Journal of Biomedical Research, vol. 2, no. 1, pp. 41-61, 2011.

[12] N. Singh, A. Pandurangan, K. Rana, P. Anand, A. Ahmad, and A. K. Tiwari, "Benzimidazole: a short review of their antimicrobial activities," International Current Pharmaceutical Journal, vol. 1, no. 5, pp. 119-127, 2012.

[13] D. K. Basedia, B. K. Dubey, and B. Shrivastava, "A review on synthesis and biological activity of heterocyclic compounds bearing 1, 3, 5-triazine lead moiety," American Journal of Pharmtech Research, vol. 1, no. 4, pp. 174-193, 2011.

[14] S. L. Khokra, K. Arora, H. Mehta, A. Aggarwal, and M. Yadav, "Common methods to synthesize benzothiazole derivatives and their medicinal significance," International Journal of Pharmaceutical Sciences and Research, vol. 2, no. 6, pp. 1356-1378, 2011.

[15] D. Shi, T. D. Bradshaw, S. Wrigley et al., "Antitumor benzothiazoles. 3. Synthesis of 2-(4-aminophenyl)benzothiazoles and evaluation of their activities against breast cancer cell lines in vitro and in vivo," Journal of Medicinal Chemistry, vol. 39, no. 17, pp. 3375-3384, 1996.

[16] S. Choi, H. J. Park, S. K. Lee, S. W. Kim, G. Han, and H. P. Choo, "Solid phase combinatorial synthesis of benzothiazoles and evaluation of topoisomerase II inhibitory activity, Bioorganic and Medicinal Chemistry, vol. 14, no. 4, pp. 1229-1235, 2006.

[17] H. A. Bhuva and S. G. Kini, "Synthesis, anticancer activity and docking of some substituted benzothiazoles as tyrosine kinase inhibitors," Journal of Molecular Graphics and Modelling, vol. 29, no. 1, pp. 32-37, 2010.

[18] V. P. Devmurari, S. Pandey, M. B. Goyani, R. R. Nandanwar, N. P. Jivani, and P. Perumal, "Phytochemical screening of ethanolic extract of Artemisia Nilagirica," International Journal of Chemical Sciences, vol. 8, no. 4, pp. 2099-2104, 2010.

[19] C. Franchini, M. Muraglia, F. Corbo et al., "Synthesis and biological evaluation of 2-mercapto-1,3-benzothiazole derivatives with potential antimicrobial activity," Archiv der Pharmazie, vol. 342, no. 10, pp. 605-613, 2009.

[20] G. Alang, R. Kaur, G. Kaur, A. Singh, and P. Singla, "Synthesis and antibacterial activity of some new benzothiazole derivatives," Acta Pharmaceutica Sciencia, vol. 52, no. 2, pp. 213-218, 2010.

[21] A. Catalano, A. Carocci, I. Defrenza et al., "2-Aminobenzothiazole derivatives: search for new antifungal agents," European Journal of Medicinal Chemistry, vol. 64, pp. 357-364, 2013.

[22] M. Mahran, S. William, F. Ramzy, and A. M. Sembel, "Synthesis and in vitro evaluation of new benzothiazole derivatives as schistosomicidal agents," Molecules, vol. 12, no. 3, pp. 622-633, 2007.

[23] P. Venkatesh and S. N. Pandeya, "Synthesis and anti-oxidant activity of some $N$-(anilinocarbonothioyl) benzamide and heterocyclic based thiourea derivatives," International Journal of ChemTech Research, vol. 1, no. 3, pp. 733-741, 2009.

[24] A. Gupta and S. Rawat, "Synthesis and anti-inflammatory study of novel fluorobenzothiazole derivatives," Journal of Chemical and Pharmaceutical Research, vol. 2, no. 5, pp. 244-258, 2010.

[25] N. D. Amnerkar and K. P. Bhusari, "Synthesis, anticonvulsant activity and 3D-QSAR study of some prop-2-eneamido and 1acetyl-pyrazolin derivatives of aminobenzothiazole," European Journal of Medicinal Chemistry, vol. 45, no. 1, pp. 149-159, 2010.

[26] N. Siddiqui, A. Rana, S. A. Khan, M. A. Bhat, and S. E. Haque, "Synthesis of benzothiazole semicarbazones as novel anticonvulsants-The role of hydrophobic domain," Bioorganic and Medicinal Chemistry Letters, vol. 17, no. 15, pp. 4178-4182, 2007.

[27] N. Siddiqui, A. Rana, S. Khan et al., "Synthesis and preliminary screening of benzothiazol-2-yl thiadiazole derivatives for anticonvulsant activity," Acta Pharmaceutica, vol. 59, no. 4, pp. 441451, 2009.

[28] G. Navarrete-Vazquez, P. Paoli, I. León-Rivera et al., "Synthesis, in vitro and computational studies of protein tyrosine phosphatase 1B inhibition of a small library of 2-arylsulfonylaminobenzothiazoles with antihyperglycemic activity," Bioorganic and Medicinal Chemistry, vol. 17, no. 9, pp. 3332-3341, 2009.

[29] A. Nitta, H. Fujii, S. Sakami et al., “(3R)-3-Amino-4-(2,4,5trifluorophenyl)-N-4-[6-(2-methoxyethoxy)benzothiazol-2yl]tetrahydropyran-4-ylbutanamide as a potent dipeptidyl peptidase IV inhibitor for the treatment of type 2 diabetes," Bioorganic and Medicinal Chemistry Letters, vol. 18, no. 20, pp. 5435-5438, 2008.

[30] P. Arora, S. Das, M. S. Ranawat, N. Arora, and M. M. Gupta, "Synthesis and biological evaluation of some novel chromene2-one derivatives for antipsychotic activity," Journal of Chemical and Pharmaceutical Research, vol. 2, no. 4, pp. 317-323, 2010.

[31] M. Anzini, A. Chelini, A. Mancini et al., "Synthesis and biological evaluation of amidine, guanidine, and thiourea derivatives 
of 2-amino-(6-trifluoromethoxy)benzothiazole as neuroprotective agents potentially useful in brain diseases," Journal of Medicinal Chemistry, vol. 53, no. 2, pp. 734-744, 2010.

[32] C. Bruno, A. Carocci, A. Catalano et al., "Facile, alternative route to lubeluzole, its enantiomer, and the racemate," Chirality, vol. 18, no. 4, pp. 227-231, 2006.

[33] M. S. Yar and Z. H. Ansari, "Synthesis and in vivo diuretic activity of biphenyl benzothiazole-2-carboxamide derivatives," Acta Poloniae Pharmaceutica, vol. 66, no. 4, pp. 387-392, 2009.

[34] D. Armenise, N. De Laurentis, A. Reho, A. Rosato, and F. Morlacchi, "Synthesis and antifungal activity against strains of Candida albicans of 6-fluoro-4(5 or 7)-chloro-2-(difluorobenzoyl)aminobenzothiazoles," Journal of Heterocyclic Chemistry, vol. 41, no. 5, pp. 771-775, 2004.

[35] D. Armenise, M. Muraglia, M. A. Florio et al., " $4 \mathrm{H}-1$, 4-benzothiazine, dihydro-1, 4-benzothiazinones and 2-amino-5-fluorobenzenethiol derivatives: design, synthesis and in vitro antimicrobial screening," Archiv der Pharmazie, vol. 345, no. 5, pp. 407-416, 2012.

[36] CLSI, Methods for Dilution Antimicrobial Susceptibility Tests for Bacteria that Grow Aerobically. Approved Standard, M7-A8, Clinical and Laboratory Standards Institute, Wayne, Pa, USA, 8th edition, 2009.

[37] CLSI, Reference Method for Broth Dilution Antifungal Susceptibility Testing of Yeast. Approved Standard, M27-A3, CLSI, Clinical and Laboratory Standards Institute, Wayne, Pa, USA, 3rd edition, 2008.

[38] C. Franchini, A. Carocci, A. Catalano et al., "Optically active mexiletine analogues as stereoselective blockers of voltagegated Na+ channels," Journal of Medicinal Chemistry, vol. 46, no. 24, pp. 5238-5248, 2003.

[39] A. Catalano, A. Carocci, G. Lentini, I. Defrenza, M. M. Cavalluzzi, and C. Franchini, "An improved synthesis of m-hydroxymexiletine, a potent mexiletine metabolite," Drug Metabolism Letters, vol. 6, no. 2, pp. 124-128, 2012.

[40] A. Catalano, A. Carocci, F. Corbo et al., "Constrained analogues of tocainide as potent skeletal muscle sodium channel blockers towards the development of antimyotonic agents," European Journal of Medicinal Chemistry, vol. 43, no. 11, pp. 2535-2540, 2008.

[41] M. M. Cavalluzzi, C. Bruno, G. Lentini et al., "One-step synthesis of homochiral O-aryl and O-heteroaryl mandelic acids and their use as efficient ${ }^{1} \mathrm{H}$ NMR chiral solvating agents," Tetrahedron Asymmetry, vol. 20, no. 17, pp. 1984-1991, 2009.

[42] A. Carocci, A. Catalano, A. Lovece et al., "Design, synthesis, and pharmacological effects of structurally simple ligands for $\mathrm{MT}_{1}$ and $\mathrm{MT}_{2}$ melatonin receptors," Bioorganic and Medicinal Chemistry, vol. 18, no. 17, pp. 6496-6511, 2010. 

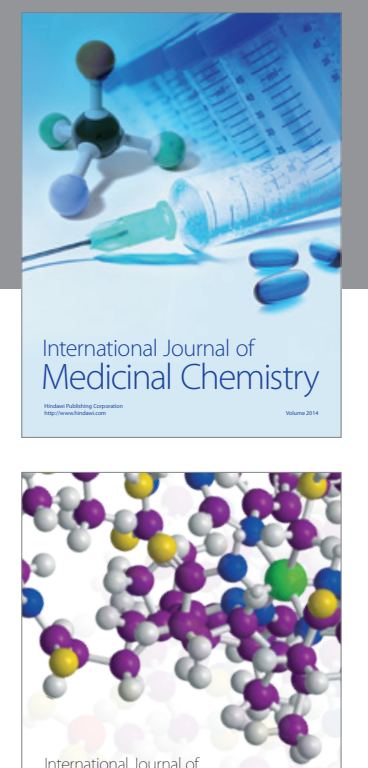

\section{Carbohydrate} Chemistry

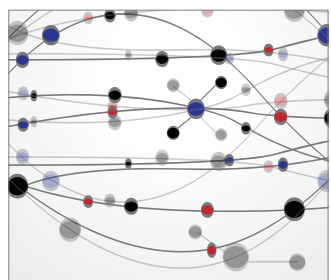

The Scientific World Journal

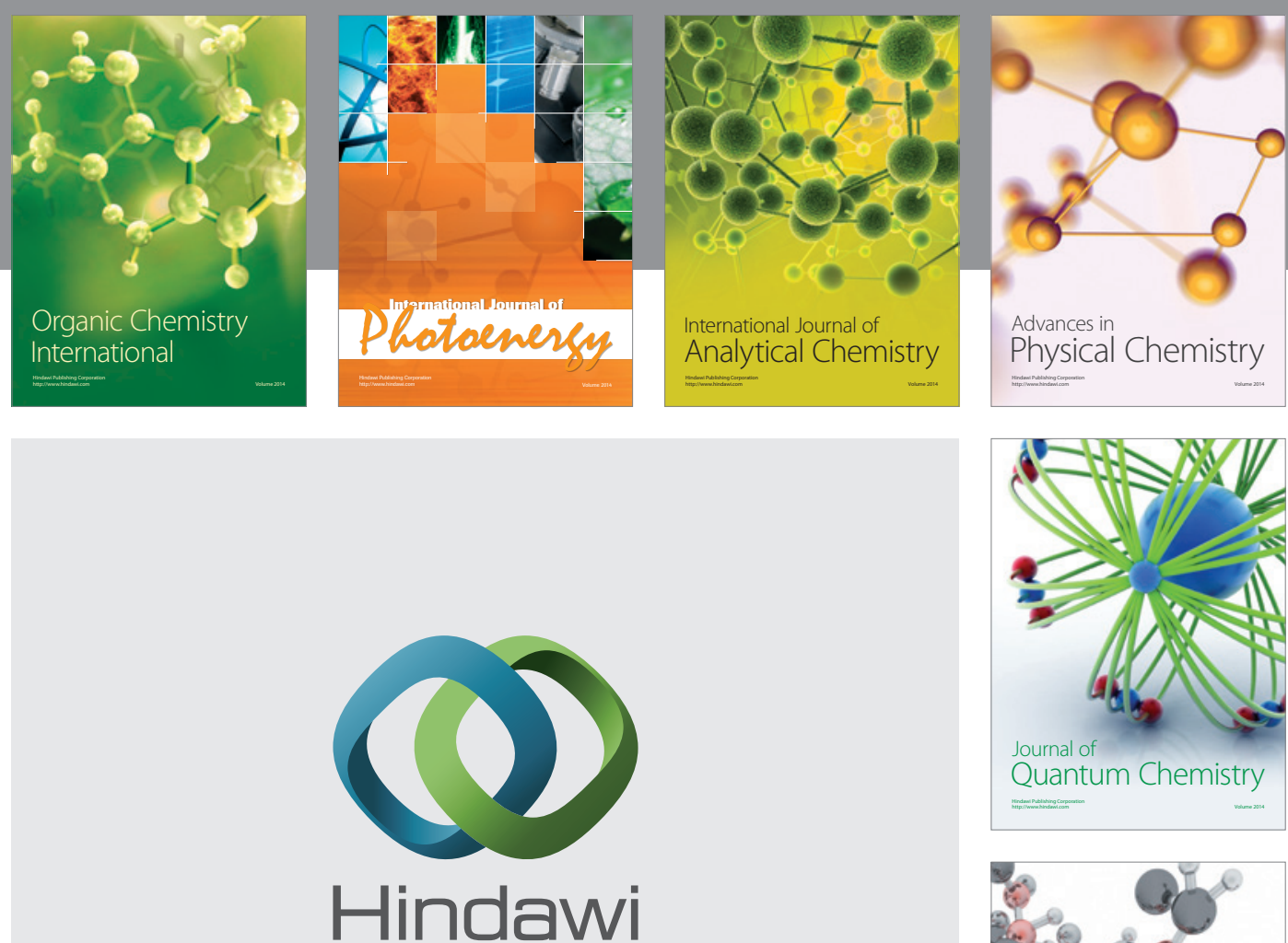

Submit your manuscripts at

http://www.hindawi.com

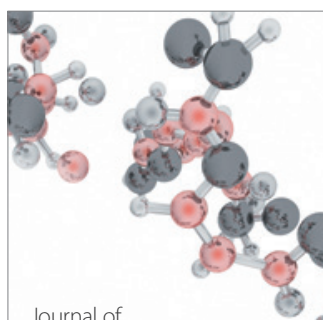

Analytical Methods

in Chemistry

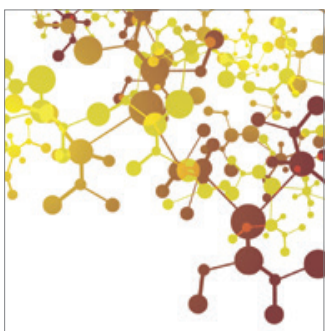

Journal of

Applied Chemistry

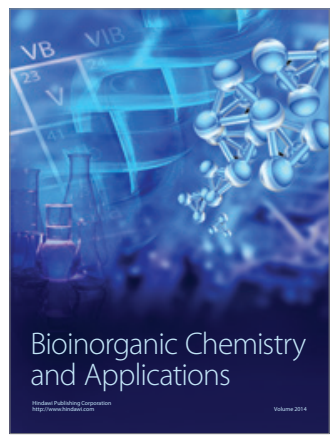

Inorganic Chemistry
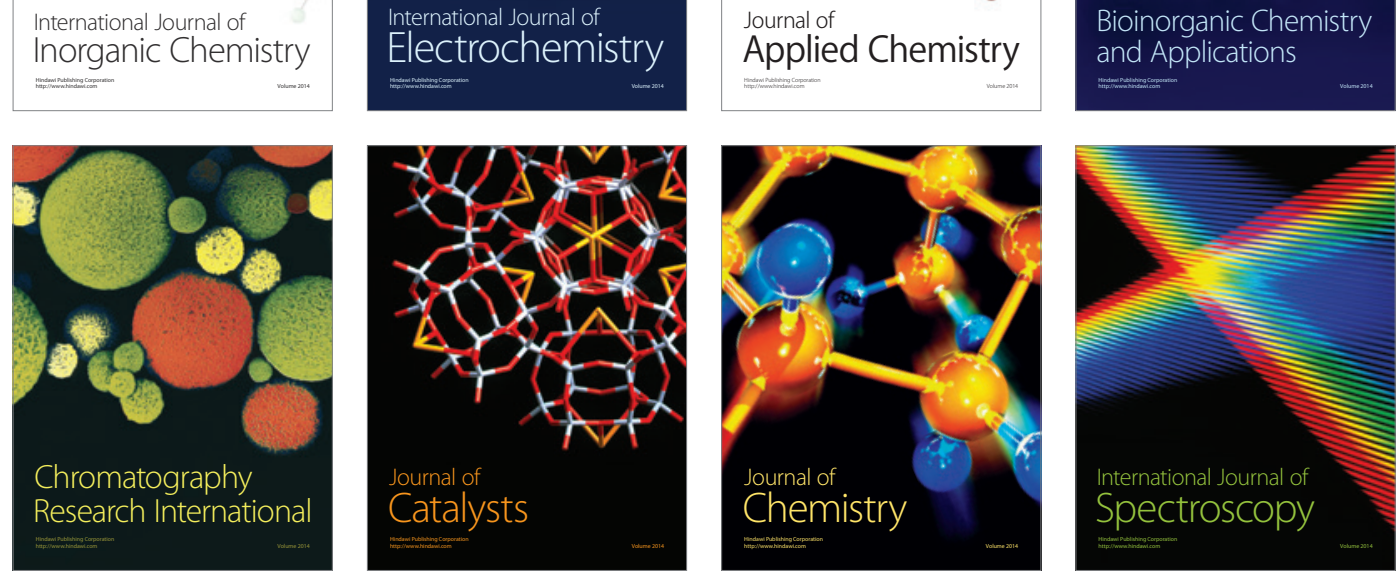